\title{
MMSE-based random sampling for iterative detection for large-scale MIMO systems
}

\author{
CHOI Jinho' ${ }^{1}$ BAI Lin ${ }^{2}$
}

1. School of Information and Communications, Gwangju Institute of Science and Technology (GIST), Gwangju 500-712, Korea

2. School of Electronic and Information Engineering, Beihang University, Beijing 100191, China

\begin{abstract}
Although large-scale MIMO can offer a high spectral efficiency, there are a number of difficulties in its implementation. Among those, the computational complexity of MIMO detection is crucial and may limit its use at devices of limited computing power such as users' mobile devices. Random sampling for large-scale MIMO detection of low complexity were studied. In particular, a MMSE approach for random sampling, was formulated from which an iterative detector can be derived for better performances.
\end{abstract}

Key words: random sampling, MMSE, large-scale MIMO

Citation: CHOI J H, BAI L. MMSE-based random sampling for iterative detection for large-scale MIMO systems[J]. Journal of communications and information networks, 2016, 1(2): 29-36

\section{Introduction}

Large-scale or massive MIMO (Multiple-Input Multiple-Output) has been considered to increase the spectral efficiency for next generation wireless systems ${ }^{[1,2]}$. In most cases, large-scale MIMO systems are considered for BSs (Base Stations). As the carrier frequency increases, the spatial correlation can be lower for a fixed antenna spacing. Thus, the physical size of antenna arrays may not be a critical issue in largescale MIMO systems of high carrier frequencies. This means that large-scale MIMO systems can also be used for mobile terminals and vehicles.

Although a high spectral efficiency can be achieved, there are various problems in large-scale MIMO systems. Among those, implementation dif- ficulties in terms of computational complexity and hardware cost would be the main obstacles in building large-scale MIMO systems. In particular, the joint signal detection in large-scale MIMO becomes computationally infeasible as the complexity grows exponentially with the number of transmit antennas. Therefore, there have been a number of approaches for low-complexity MIMO detection methods. Linear detectors can be considered as they have low computational complexities ${ }^{[3,4]}$. It is also possible to formulate the MIMO detection problem as a convex optimization problem and use SDR (Semi Definite Relaxation) to find an approximate solution to the ML(Maximum Likelihood) detection problem ${ }^{[5]}$. Lattice basis reduction has been applied to MIMO $\operatorname{detection}^{[4,6,7]}$. 
These well known approaches have their own drawbacks. For example, linear detectors, e.g., ZF (Zero-Forcing) and MMSE(Minimum Mean Squared Error) detectors, have poor performances (especially, at high SNR(Signal-to-Noise Ratio)). The complexity of lattice basis reduction is prohibitively high when the number of transmit antennas is large. SDR approximation also has a similar complexity problem for large-scale MIMO.

In this paper, we consider an approach that can provide better performances than linear detectors using random sampling. This approach is not only computationally efficient (as it is based on linear detectors), but also easy to perform iterations for better performances using the MMSE formulation.

The rest of the paper is organized as follows. A system model for the MIMO detection is presented in Section 2. A list detection method with random sampling is explained in Section 3. A random sampling method based on MMSE formulation are proposed with an iterative detector in Section 4. A performance analysis is presented in Section 5 and simulation results are given in Section 6. Some remarks are conducd in Section 7.

Notation: Matrices and vectors are denoted by upper- and lower-case boldface letters, respectively. The superscripts $\mathrm{T}$ and $\mathrm{H}$ denote the transpose and complex conjugate, respectively. The 2-norm of $\boldsymbol{a}$ is denoted by $\|\boldsymbol{a}\|$. For a given vector, $\boldsymbol{x},[\boldsymbol{x}]_{m}$ denotes the $m$-th element. For a matrix, $\boldsymbol{X},[\boldsymbol{X}]_{m, n}$ represents the $(m$, $n)$-th element. $\mathbb{E}[\cdot]$ denotes the statistical expectation. $\mathcal{C N}(\boldsymbol{a}, \boldsymbol{R})$ represents the distribution of CSCG (Circularly Symmetric Complex Gaussian) random vectors with mean vector $\boldsymbol{a}$ and covariance matrix $\boldsymbol{R}$.

\section{System model}

Suppose that a transmitter is equipped with $K$ antennas and a receiver is equipped with $N$ antennas. Denote by $s_{k}$ the symbol to be transmitted by transmit antenna $k$. We assume that $s_{k} \in \mathcal{S}$ for all $k$, where $\mathcal{S}$ represents the signal constellation. In addition, we assume that the mean and variance of $s_{k}$ are 0 and $E_{\mathrm{S}}$, respectively. Here, $E_{S}$ denotes the symbol energy. For $M$-ary signaling, we have $|\mathcal{S}|=M$. Let $\boldsymbol{s}=\left[s_{1} \ldots s_{K}\right]^{\mathrm{T}}$. In addition, denote by $r_{q}$ and $n_{q}$ the received signal and background noise at receive antenna $q$, respectively. Then, we have

$$
\begin{aligned}
\boldsymbol{r} & =\left[\begin{array}{lll}
r_{1} & \cdots & r_{N}
\end{array}\right]^{\mathrm{T}} \\
& =\boldsymbol{H} \boldsymbol{s}+\boldsymbol{n},
\end{aligned}
$$

where $\boldsymbol{n}=\left[n_{1} \ldots n_{N}\right]^{\mathrm{T}}$. Here, $\boldsymbol{H}$ denotes the channel matrix from the transmitter to the receiver, where $\lfloor\boldsymbol{H}\rfloor_{n, k}$ represents the channel coefficient from transmit antenna $k$ to receive antenna $n$. Throughout the paper, $\boldsymbol{n} \sim \mathcal{C N}\left(0, N_{0} \boldsymbol{I}\right)$, i.e., the background noise vector is assumed to be zero-mean CSCG random vector.

For convenience, we convert the complex-valued channel model into the real-valued one. Let

$$
\boldsymbol{H}=\left[\begin{array}{ll}
\mathfrak{R}(\boldsymbol{H}) & \mathfrak{I}(\boldsymbol{H}) \\
\mathfrak{I}(\boldsymbol{H}) & \mathfrak{R}(\boldsymbol{H})
\end{array}\right]
$$

In addition, let

$$
\boldsymbol{r}=\left[\begin{array}{c}
\mathfrak{R}(r) \\
\mathfrak{I}(r)
\end{array}\right], \boldsymbol{s}=\left[\begin{array}{c}
\mathfrak{R}(s) \\
\mathfrak{I}(s)
\end{array}\right] \text {, and } \boldsymbol{n}=\left[\begin{array}{c}
\mathfrak{R}(n) \\
\mathfrak{I}(n)
\end{array}\right] .
$$

Then, we have

$$
\boldsymbol{r}=\boldsymbol{H} \boldsymbol{s}+\boldsymbol{n} .
$$

For convenience, we assume that each element of $s$ is an integer, i.e., $[s]_{k} \in \mathbb{Z}$, where $\mathbb{Z}$ denotes the set of integers, after proper scaling and shifting ${ }^{[4]}$.

\section{List detection using random sam- pling}

An optimal detection can be carried out using the ML criterion. That is,

$$
\hat{s}_{\mathrm{m} 1}=\arg \max _{\boldsymbol{s} \in S^{K}} f(\boldsymbol{r} \mid \boldsymbol{s}),
$$

where $f(\boldsymbol{r} \mid \boldsymbol{s})$ is the likelihood function of $\boldsymbol{s}$ for given $\boldsymbol{r}$. Since $\left|\mathcal{S}^{K}\right|$ is $M^{K}$, we can see that the complexity of the ML detection grows exponentially with $K$ if an exhaustive search is used. This shows that the ML de- 
tection is computationally infeasible for a large $K$.

We slightly modify the ML detection in Eq.(3) as

$$
\begin{aligned}
\hat{s} & =\arg \max _{\boldsymbol{s} \in \mathbb{Z}^{2 K}} f(\boldsymbol{r} \mid \boldsymbol{s}), \\
& =\arg \max _{\boldsymbol{s} \in \mathbb{Z}^{2 K}}\|\boldsymbol{r}-\boldsymbol{H} \boldsymbol{s}\|^{2} .
\end{aligned}
$$

The signal space, $\mathcal{S}^{K}$, is now expanded to $\mathbb{E}^{2 K}$. Since it is not possible to find $\hat{s}$ that minimizes the likelihood function from $\mathbb{Z}^{2 K}$, we may consider a list of candidate signal vectors of finite length. Denote by $\mathcal{I}$ the list. If we are able to draw samples from $f(\boldsymbol{r} \mid \boldsymbol{s})$ (assuming $f(\boldsymbol{r} \mid \boldsymbol{s})$ as a distribution), it is possible to build a list of candidate vectors from sampling. In this list, we can expect to have the ML solution with a high probability since $\hat{\boldsymbol{s}}$, i.e., the ML solution, is the sample of the largest likelihood value.

In Refs.[8,9], MCMC (Markov chain Monte Carlo) algorithms ${ }^{[10]}$ are considered to build a list from random sampling. However, this approach has a drawback. In order to find a list of good candidate vectors, it requires a long burn-in time or a number of iterations. This makes MCMC algorithms less attractive if the receiver has limited computing power such as mobile terminals.

As an alternative, we can consider the approach proposed in Ref.[11] for random sampling to build a list, which has been used for MIMO detection in Refs.[12,13] with lattice basis reduction. However, since the complexity of lattice basis reduction can be prohibitively high for a large number of transmit antennas ${ }^{[14]}, K$, this approach may not be suitable for large-scale MIMO systems. Fortunately, we can have random samples based on this approach without lattice basis reduction at the cost of performance degradation.

For convenience, we assume that $N \geqslant K$ and consider the $Q R$ factorization of $\boldsymbol{H}$ as

$$
\boldsymbol{H}=\boldsymbol{Q} \boldsymbol{R},
$$

where $\boldsymbol{Q}$ and $\boldsymbol{R}$ are unitary and upper triangular, respectively. Let $\bar{K}=2 K$ and $\bar{N}=2 N$. Clearly, the size of $\boldsymbol{H}$ is $\bar{N} \times \bar{K}$. Let

$$
\boldsymbol{x}=\boldsymbol{Q}^{\mathrm{H}} \boldsymbol{r} .
$$

If we consider the SIC (Successive Interference Cancellation) approach for detection ${ }^{[4]}$, it follows

$$
\hat{s}=\text { Round }\left(\frac{x_{k}-\sum_{q=k+1}^{\bar{K}} r_{k, q} \hat{s}_{q}}{r_{k, k}}\right), k=\bar{K}, \cdots, 1,
$$

where $\operatorname{Round}(x)$ is the round operation to convert $x$ into an integer. This rounding operation can be replaced by a randomized rounding operation that is associated with a certain distribution in order to generate a list of candidate symbol vectors. Define the following distribution, which is a Gaussian-like distribution:

$$
\operatorname{Pr}(Q=q ; \psi)=\frac{\mathrm{e}^{-\psi(r-q)^{2}}}{s},
$$

where $\psi>0$ a parameter that decides the degree of randomness and

$$
S=\sum_{q=-\infty}^{\infty} \mathrm{e}^{-\psi(r-q)^{2}} .
$$

For any $r$, we can show that

$$
r-q=\lfloor r\rfloor+\epsilon-q,
$$

where $0<\epsilon \triangleq r-\lfloor r\rfloor<1$. From this, it can be shown that

$$
\begin{aligned}
& s=\sum_{q=-\infty}^{\infty} \mathrm{e}^{-\psi([r]-q+\epsilon)^{2}} \\
& \leqslant s(\psi) \triangleq \sum_{q=-\infty}^{\infty} \mathrm{e}^{-\psi q^{2}}=\sum_{q=0}^{\infty} \mathrm{e}^{-\psi q^{2}}+\sum_{q=1}^{\infty} \mathrm{e}^{-\psi(q+1)^{2}},
\end{aligned}
$$

where $s(\psi)$ is an upper-bound on $s$, which is independent of $r$.

With the distribution in Eq.(5), the randomized rounding can be defined as randRound $_{\nu}(x)=q$, with probability $\operatorname{Pr}(Q=q ; x), q \in \mathrm{Z}$

The randomized rounding becomes the deterministic rounding if $\psi$ approaches $\infty$. Using the randomized rounding, the randomized SIC detection is given by

$\hat{\boldsymbol{s}}_{k}=$ sandRound $_{\psi_{k}}\left(\frac{x_{k}-\sum_{q=k+1}^{\bar{K}} r_{k, q} \hat{s}_{q}}{r_{k, k}}\right), k=\bar{K}, \cdots, 1$,

where

$$
\psi_{k}=A\left|r_{k, k}\right|^{2} .
$$


Here, $A>0$ is a design parameter that plays a crucial role in providing a good performance. This randomized SIC detection can provide a different hard-decision of $s$ for each call, which is an important feature to build a list. With $L_{\text {list }}$ calls, we can have a list of $L_{\text {list }}$ candidate vectors (note that there might be some duplicate candidate vectors). With this list, the best vector that maximizes the likelihood function can be found for the list detection. Thus, the performance can be improved as $L_{\text {list }}$ increases, while the complexity grows linearly. In Ref.[12], an approach to decide $A$ in Eq.(9) is derived. Note that due to possible presence of duplicate candidate vectors, the increase of $L_{\text {list }}$ does not necessarily result in an improved performance.

Throughout this paper, the detection method in Eq.(8) is referred to as the ZF-based detector with random sampling as the interference signals not yet canceled are suppressed. This approach can have good performances as samples are obtained from the likelihood function. To see this, from Eq.(8), we can derive the probability of $s$ as

$$
\begin{aligned}
\operatorname{Pr}(\hat{\boldsymbol{s}}) & =\prod_{k=1}^{\bar{K}} \operatorname{Pr}\left(\hat{\boldsymbol{s}}_{k}\right)=\prod_{k=1}^{\bar{K}} \frac{1}{A_{k}} \mathrm{e}^{-\psi_{k}\left(z_{k}-\hat{s}_{k}\right)^{2}} \\
& =\left(\prod_{k=1}^{\bar{K}} \frac{1}{A_{k}}\right) \mathrm{e}^{-A\|\boldsymbol{x}-\boldsymbol{R} \boldsymbol{s}\|^{2}},
\end{aligned}
$$

where $A_{k}$ is the normalization factor and

$$
z_{k}=\frac{x_{k}-\sum_{q=k+1}^{\bar{K}} \hat{s}_{k}}{r_{k, k}} \text {. }
$$

Thus, if $A=\frac{1}{N_{0}}$, we can see that

$$
\operatorname{Pr}(\hat{\boldsymbol{s}}) \propto f(\boldsymbol{r} \mid \hat{\boldsymbol{s}}) .
$$

While this approach can provide good performances, there are a couple of difficulties. This approach may not be applicable to the case where $N<K$ (overloaded systems). In addition, no prior information can be incorporated, which is important to derive an iterative approach. In the next section, we modify the ZF-based detector with random sampling using the
MMSE formulation to overcome the above difficul-ties.

\section{MMSE approach with gaussian ap- proximation}

In this section, we employ the MMSE formulation to modify the ZF-based detector with random sampling. This formulation allows to derive an iterative detection method.

Suppose that the a priori distribution of $s$ is available as

$$
\boldsymbol{s} \sim \mathcal{N}\left(\overline{\boldsymbol{s}}_{\mathrm{I}}, \boldsymbol{C}_{\mathrm{I}}\right),
$$

where $\mathbb{E}[\boldsymbol{s}]=\overline{\boldsymbol{s}}_{\mathrm{I}}$ and $\operatorname{Cov}(\boldsymbol{s})=\boldsymbol{C}_{\mathrm{I}}$, which is an approximation as $s$ is a discrete random vector. However, this Gaussian approximation allows us to adopt the MMSE formulation easily. Then, it can be shown that ${ }^{[4,15]}$

$$
\boldsymbol{g}=\left[\begin{array}{l}
\boldsymbol{s} \\
\boldsymbol{r}
\end{array}\right] \sim \mathcal{N}\left(\left[\begin{array}{l}
\overline{\boldsymbol{s}}_{\mathrm{I}} \\
\boldsymbol{H} \overline{\boldsymbol{s}}_{\mathrm{I}}
\end{array}\right],\left[\begin{array}{lc}
\boldsymbol{C}_{\mathrm{I}} & \boldsymbol{C}_{\mathrm{I}} \boldsymbol{H}^{\mathrm{T}} \\
\boldsymbol{H} \boldsymbol{C}_{\mathrm{I}} & \boldsymbol{H} \boldsymbol{C}_{\mathrm{I}} \boldsymbol{H}^{\mathrm{T}}+\sigma^{2} \boldsymbol{I}
\end{array}\right]\right),
$$

We can derive the conditional probability density function (pdf) of $\boldsymbol{s}$ for given $\boldsymbol{r}$ as follows:

$$
\boldsymbol{s} \mid \boldsymbol{r} \sim \mathcal{N}(\overline{\boldsymbol{s}}, \boldsymbol{C}),
$$

where

$$
\begin{aligned}
& \overline{\boldsymbol{s}}=\overline{\boldsymbol{s}}_{\mathrm{I}}+\boldsymbol{C}_{\mathrm{I}} \boldsymbol{H}^{\mathrm{T}}\left(\boldsymbol{H} \boldsymbol{C}_{\mathrm{I}} \boldsymbol{H}^{\mathrm{T}}+\sigma^{2} \mathbf{I}\right)^{-1}\left(\boldsymbol{r}-\boldsymbol{H}_{\mathrm{s}}\right) \\
& \boldsymbol{C}=\boldsymbol{C}_{\mathrm{I}}-\boldsymbol{C}_{\mathrm{I}} \boldsymbol{H}^{\mathrm{T}}\left(\boldsymbol{H} \boldsymbol{C}_{\mathrm{I}} \boldsymbol{H}^{\mathrm{T}}+\sigma^{2} \mathbf{I}\right)^{-1} \boldsymbol{H} \boldsymbol{C}_{\mathrm{I}},
\end{aligned}
$$

Thus, the a posteriori pdf of $s$ is given by

$$
\operatorname{Pr}(\boldsymbol{s} \mid \boldsymbol{r})=C \exp \left(-\frac{1}{2}(\boldsymbol{s}-\overline{\boldsymbol{s}})^{\mathrm{T}} \boldsymbol{C}^{-1}(\boldsymbol{s}-\overline{\boldsymbol{s}})\right),
$$

We now want to draw samples from $\operatorname{Pr}(\boldsymbol{s} \mid \boldsymbol{r})$.

Consider the Cholesky factorization of $\boldsymbol{C}^{-1}$ as

$$
\frac{1}{2} \boldsymbol{C}^{-1}=\boldsymbol{L L}^{\mathrm{T}} .
$$

Then, it follows

$$
\operatorname{Pr}(\boldsymbol{s} \mid \boldsymbol{r})=C \exp \left(-\|\boldsymbol{U}(\boldsymbol{s}-\overline{\boldsymbol{s}})\|^{2}\right),
$$

where $\boldsymbol{s} \in \mathcal{S}^{K}$ and $\boldsymbol{U}=\boldsymbol{L}^{\mathrm{T}}$. Noting that $\boldsymbol{U}$ is upper-triangular, using Eq.(15), we can find a sample of $s_{k}$ drawn from $\operatorname{Pr}(\boldsymbol{s} \mid \boldsymbol{r})$, which is denoted by $\bar{s}_{k}$. Let 


$$
x_{k}=\bar{s}_{k}-\sum_{n=k+1}^{K} \frac{u_{k, n}}{u_{k, k}}\left(s_{n}-\bar{s}_{n}\right)
$$

and

$$
\operatorname{Pr}\left(s_{k}=q\right)=\frac{1}{A_{k}} \exp \left(-\psi_{k}\left(x_{k}-q\right)^{2}\right),
$$

where $\psi_{k}=\alpha\left|u_{k, k}\right|^{2}$ and $\alpha$ is a design parameter. Then, as in Section 3, we can find a list of samples from $\operatorname{Pr}(\boldsymbol{s} \mid \boldsymbol{r})$.

It is noteworthy that the inverse of $C$ in Eq.(14) can be found easily. Using the matrix inversion lemma, we have

$$
\boldsymbol{C}^{-1}=\boldsymbol{C}_{\mathrm{I}}^{-1}+\sigma^{2} \boldsymbol{H}^{\mathrm{T}} \boldsymbol{H}
$$

Thus, we only need to perform the inverse of $\boldsymbol{C}_{\mathrm{I}}$. If the elements of $\boldsymbol{s}$ are assumed to be independent, $\boldsymbol{C}_{\mathrm{I}}$ become diagonal matrix. Thus, the complexity to obtain $\boldsymbol{C}^{-1}$ is low. The resulting approach is referred to as the MMSE-based detector with random sampling.

Using the MMSE formulation with Gaussian approximation, we can derive an iterative detection method. At the $j$ th iteration, the mean and covariance of $s$ can be found from the list of the previous iteration, say the $(j-1)$ th iteration. In each iteration, we can have a better prior information, which can result in better sampled candidate vectors. Let $\mathcal{I}_{\mathrm{j}}$ denote the list of candidate vectors from the $j$ th iteration. Then, the mean vector and covariance matrix of $s$ from the a priori distribution for the $j$ th iteration are given by

$$
\overline{\boldsymbol{s}}_{\mathrm{I}}=\sum_{\boldsymbol{s} \in \mathcal{I}_{j-1}} \boldsymbol{s},
$$

and

$$
\boldsymbol{C}_{\mathrm{I}}=\sum_{\boldsymbol{s} \in \mathcal{I}_{j-1}} \boldsymbol{s} \boldsymbol{s}^{\mathrm{T}}-\overline{\boldsymbol{s}}_{\mathrm{I}} \overline{\boldsymbol{s}}_{\mathrm{I}}^{\mathrm{H}},
$$

respectively. Note that the initial mean vector and co_ variance matrix are $\mathbf{0}$ and $\frac{E_{s}}{2} \mathbf{I}$, respectively. At the $j$ th iteration, a total list of candidate vectors is given as

$$
\overline{\mathcal{I}}_{j}=\bigcup_{i=1}^{j} \mathcal{I}_{i} \text {. }
$$

Thus, the length of this list is $j L_{\text {list }}$. If we consider $J$ iterations, the length becomes $J L_{\text {list. }}$ Using this iterative detection method, we expect to have a better list than a list of the same length that can be obtained without iteration.

\section{Performance analysis}

In this section, we consider the performance of the MMSE-based detector with random sampling with the decoding radius.

The squared decoding radius ${ }^{[12]}$ can be found as

$$
\begin{aligned}
R_{d}^{2} & =\|\boldsymbol{U}(\boldsymbol{s}-\overline{\boldsymbol{s}})\|^{2} \\
& \propto \min _{k}\left|u_{k, k}\right|^{2},
\end{aligned}
$$

We have the following result.

Theorem 1 For any Hermitian psd matrix $\boldsymbol{C}$, we have

$$
\min _{k}\left|u_{k, k}\right|^{2} \geqslant \frac{1}{\lambda \max (\boldsymbol{C})} .
$$

Proof 1 Consider the LDL factorization of $\boldsymbol{C}^{-1}$ as follows:

$$
\boldsymbol{C}^{-1}=\overline{\boldsymbol{L}} \boldsymbol{D} \overline{\boldsymbol{L}}^{\mathrm{T}},
$$

where $\bar{L}$ is lower unitriangular (the diagonal elements are all 1) and $\boldsymbol{D}$ is diagonal (as $\boldsymbol{C}$ is Hermitian psd, the diagonal elements of $\boldsymbol{D}$ are nonnegative). Clearly, we have $\boldsymbol{L}=\overline{\boldsymbol{L}} \boldsymbol{D}^{\frac{1}{2}}$ and

$$
\min _{k}\left|u_{k, k}\right|^{2}=\min _{k}[\boldsymbol{D}]_{k, k} .
$$

To find $\min _{k}\left|u_{k, k}\right|^{2}$, we can formulate the following optimization problem:

$$
\min _{k}\left|u_{k, k}\right|^{2}=\min _{k} \boldsymbol{e}_{k}^{\mathrm{T}} \overline{\boldsymbol{L}}^{-1} \boldsymbol{C}^{-1} \overline{\boldsymbol{L}}^{-\mathrm{T}} \boldsymbol{e}_{k},
$$

where $\boldsymbol{e}_{k}$ is the standard basis vector whose elements are all zero except the $k$ th element which is one. Since $\overline{\boldsymbol{L}}$ is lower unitriangular, $\overline{\boldsymbol{L}}^{-\mathrm{T}}$ is upper unitriangular and $\boldsymbol{L}^{-\mathrm{T}} \boldsymbol{e}_{k}$ is the $k$ th column vector of $\boldsymbol{L}^{-\mathrm{T}}$, in which the $k$ th element is 1 . This implies that

$$
\left\|\boldsymbol{L}^{-\mathrm{T}} \boldsymbol{e}_{k}\right\| \geqslant 1 \text {. }
$$

Then, since

$$
\left\{\boldsymbol{L}^{-\mathrm{T}} \boldsymbol{e}_{k}, k=1, \cdots, K\right\} \subset\{\boldsymbol{x} \mid\|\boldsymbol{x}\| \geqslant 1\},
$$

we have the following crucial observation:

$$
\min _{k} \boldsymbol{e}_{k}^{\mathrm{T}} \overline{\boldsymbol{L}}^{-1} \boldsymbol{C}^{-1} \overline{\boldsymbol{L}}^{-\mathrm{T}} \boldsymbol{e}_{k} \geqslant \min _{\|x\| \geqslant 1} \boldsymbol{x}^{\mathrm{T}} \boldsymbol{C}^{-1} \boldsymbol{x} .
$$

From Eq.(23), it follows that 


$$
\min _{k}\left|u_{k, k}\right|^{2} \geqslant=\min _{\|x\| \geqslant 1} \boldsymbol{x}^{\mathrm{T}} \boldsymbol{C}^{-1} \boldsymbol{x}=\lambda_{\min }\left(\boldsymbol{C}^{-1}\right),
$$

which implies Eq.(20). This completes the proof.

Since

$$
\frac{1}{\lambda_{\max }(\boldsymbol{C})} \lambda_{\min }\left(\boldsymbol{C}^{-1}\right)=\lambda_{\min }\left(\boldsymbol{C}_{\mathrm{I}}^{-1}+\sigma^{2} \boldsymbol{H}^{\mathrm{T}} \boldsymbol{H}\right)
$$

we can see that the decoding radius, $R_{d}$ can be greater than 0 even if $\bar{K}>\bar{N}$. Note that for the ZF-based detector with random sampling in Section 3 has the squared decoding radius from Eq.(10) as follows:

$$
R_{\mathrm{d}}^{2}=\left\|\boldsymbol{R}\left(\boldsymbol{R}^{-1} \boldsymbol{x}-\hat{\boldsymbol{s}}\right)\right\|^{2} \propto \min _{k}\left|r_{k, k}\right|^{2} .
$$

This squared decoding radius is lower-bounded as

$$
\min _{k}\left|r_{k, k}\right|^{2} \geqslant \lambda_{\text {min }}\left(\boldsymbol{H}^{\mathrm{T}} \boldsymbol{H}\right) .
$$

If $\bar{K}>\bar{N}, \lambda_{\min }\left(\boldsymbol{H}^{\mathrm{T}} \boldsymbol{H}\right)=0$. Thus, the decoding radius can be 0 , which implies that the ZF-based detector with random sampling cannot be applied to the case of $\bar{K}>\bar{N}$, while the MMSE-based detector with random sampling is applicable.

Furthermore, from Eq.(20), we can see that if prior information is more reliable (i.e., the diagonal elements of $\boldsymbol{C}_{\mathrm{I}}$ are close to zeros), the diagonal elements of $\boldsymbol{C}_{\mathrm{I}}^{-1}$ are larger, which makes the lower-bound larger and results in better performances.

\section{Simula}

In this section, we present simulation results with $M$-ary quadrature amplitude modulation (QAM) signaling for a class of MIMO channels where $[\boldsymbol{m} \boldsymbol{H}]_{n, k} \sim \mathcal{C N}(0,1 / N)$. The SNR is $E_{\mathrm{b}} / N_{0}$, where $E_{\mathrm{b}}$ is the bit energy.

Fig.1 shows the BER (Bit Error Rate) performances of the three detectors: (i) the MMSE detector (without random sampling), (ii) the ZF-based detector with random sampling, (iii) the MMSE-based detector with random sampling. We can see that the list detectors with random sampling (i.e., (ii) and (iii)) perform better than the MMSE detector. In particular, at a low BER (less than $10^{-2}$ ), we can have about 5
dB SNR gain by using random sampling. It is shown that both the ZF-based and MMSE-based detectors with random sampling have similar performances. In the MMSE-based detector with random sampling, we assume that the prior information is initialized as follows:

$$
s \sim \mathcal{N}\left(\mathbf{0}, \frac{E_{s}}{2} \mathbf{I}\right),
$$

where $E_{s}$ is the (complex-valued) symbol energy, i.e., $E_{s}=\mathbb{E}\left[\left|s_{k}\right|^{2}\right]$. Thus, no particular prior information that can help improve the performance is used in the MMSE-based detector with random sampling.

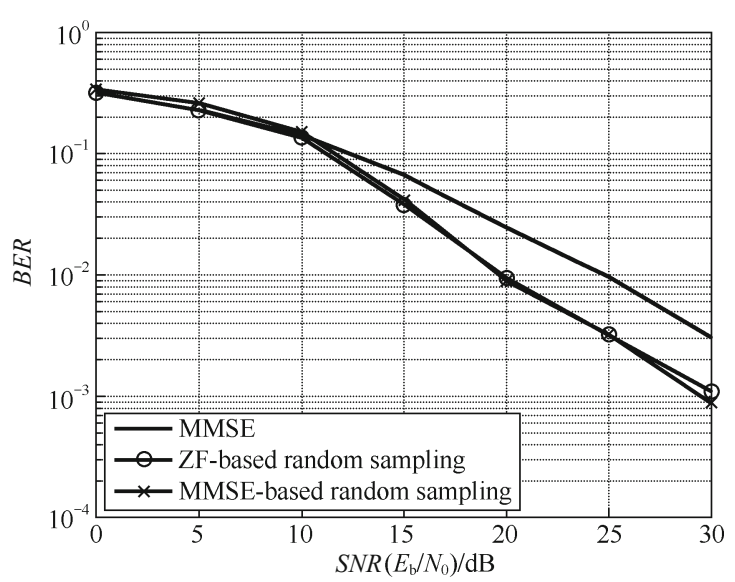

Figure $1 B E R$ versus $S N R$ when $N=K=10$ and 16QAM is used. For random sampling, the length of the list is $L_{\text {list }}=2 K=20$

As discussed in Section 4, the MMSE formulation allows us to incorporate prior information if available. Thus, we can build an iterative detector, where the list of candidate vectors of the previous iteration is used as prior information. Fig. 2 shows the performances of this iterative detector when $N=K=20$ and 4-QAM is used. In each iteration, the MMSE-based detector with random sampling provides a list of $L_{\text {list }}=20$ candidate vectors. The length of the overall list increases with iterations by adding new list at each iteration. Thus, after 6 iterations, we have a list of length $6 \times L_{\text {list }}=20$. We can see that the performance can be improved by iterations. In Fig.2, we also have BER 
performance of the list detection without iteration when the list length is set to 120 . This detection has a slightly better performance than that of the iterative detector after one iteration (whose list length is 20). Consequently, we can observe that the performance may not be significantly improved with a longer list unless good prior information is provided. In order to improve the performance further, the iterative detection method can be employed as better prior information can be exploited through iterations.

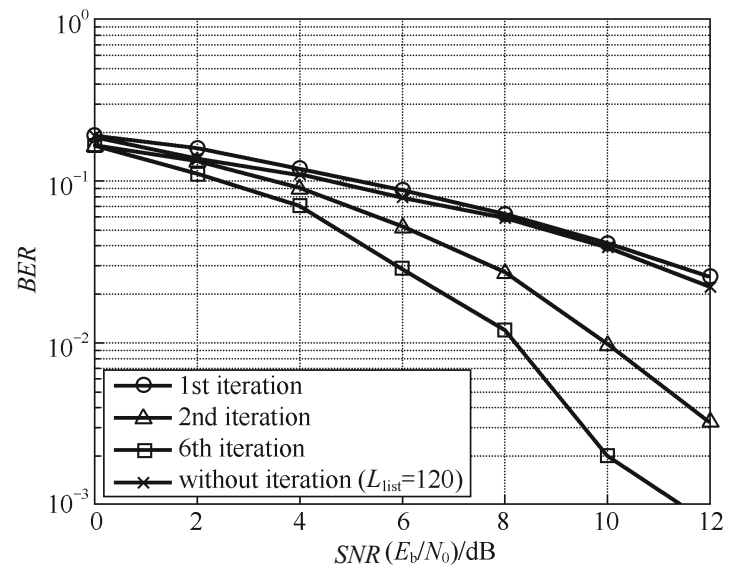

Figure 2 BER performances of the MMSE-based iterative detector with random sampling when $N=K=20$ and 4-QAM is used. In each iteration, the length of the list is $L_{\text {list }}=20$

In order to see the impact of iterations, we show the BER curves as functions of iterations when [Trial mode] and 16-QAM is used in Fig. 3. It is shown that the performance cannot be improved after a few iterations, i.e., there is error floor. As a further research issue, we could consider an analysis to derive the error floor or performance limits.

As mentioned earlier, an advantage of the MMSEbased iterative detector with random sampling over the ZF-based iterative detector with random sampling is that it can be used when $K>N$. We consider simulations with various values of $K$ and present the results in Figure 4 with $N=10$ and 4-QAM. It is shown that the performance the MMSE-based iterative de- tector with random sampling is better than that of the MMSE detector, and the performance gap increases as $K$ decreases.

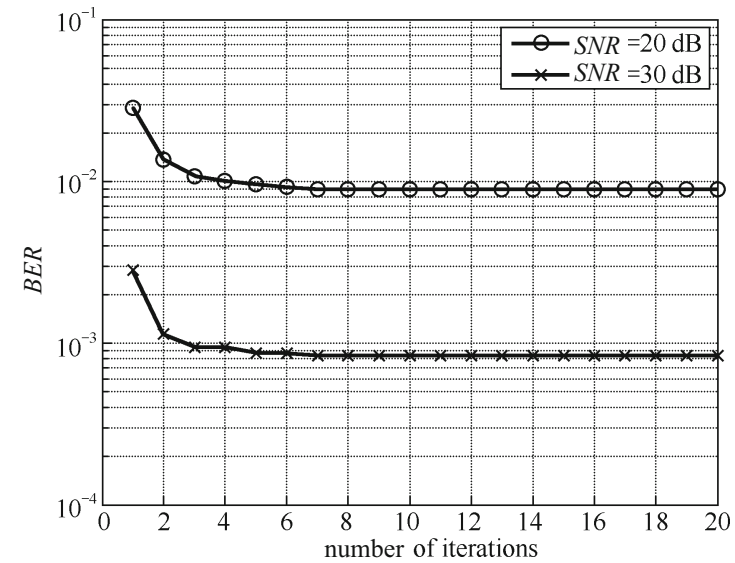

Figure 3 BER versus iterations of the MMSE-based iterative detector with random sampling when $N=K=4$ and 16-QAM is used. For random sampling, the length of the list for each iteration is $L_{\text {list }}=20$

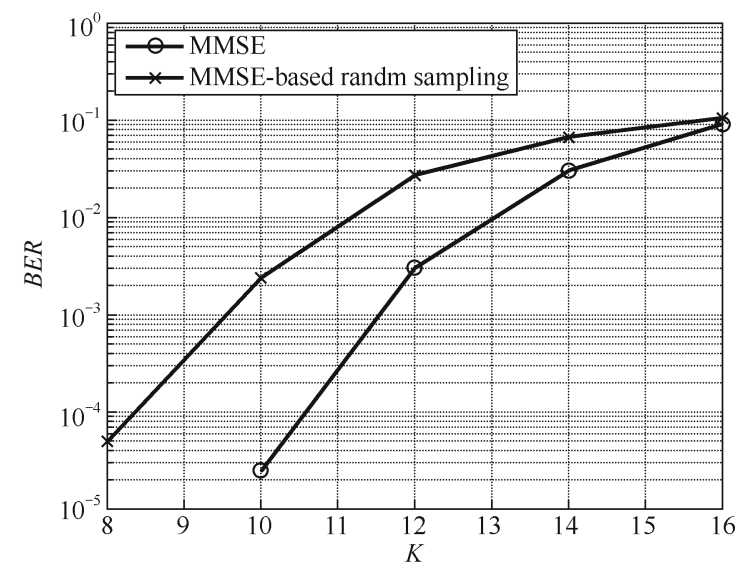

Figure 4 BER performances of the MMSE detector and MMSE-based iterative detector with random sampling for different numbers of transmit antennas, $K$, when $S N R=20 \mathrm{~dB}, N=10$, and 4-QAM is used. For random sampling, the length of the list is $L_{\text {list }}=4 K$.

\section{Conclusion}

In this paper, we studied list detection using random sampling. By adopting the MMSE formulation, it was 
possible to derive an iterative detector, which can improve the performance by iterations as better prior information could be available. It was shown that the resulting detector has better performance than a linear detector (i.e., the MMSE detector). Furthermore, since it was based on the MMSE formulation, it can be used for overloaded systems (i.e., more transmit antennas than receive antennas).

\section{References}

[1] RUSEK F, PERSSON D, LAU B K, et al. Scaling up mimo: Opportunities and challenges with very large arrays[J]. IEEE signal processing magazine, 2013, 30(1): 40-60.

[2] LARSSON E, EDFORS O, TUFVESSON F, et al. Massive MIMO for next generation wireless systems[J]. IEEE communications magazine, 2014, 52(2): 186-195.

[3] VERDU S. Multiuser detection[M]. Cambridge: Cambridge University Press, 1998.

[4] CHOI J. Optimal combining and detection[M]. Cambridge: Cambridge University Press, 2010.

[5] PALOMAR D P, ELDAR Y C. Convex optimization in signal processing and communications[M]. Cambridge: Cambridge University Press, 2009.
[6] YAO H, WORNELL G W. Lattice-reduction-aided detectors for MIMO communication systems[C]//Proc of IEEE GLOBCCOM, 2002: 17-21.

[7] WUBBEN D, BOHNKE R, KUHN V, et al. Near-maximum-likelihood detection of MIMO systems using MMSE-based lattice reduction[C]//Proc of ICC, Paris, France, 2004: 798-802.

[8] CHEN R, LIU J, WANG X. Convergence analyses and comparisons of markov chain monte carlo algorithms in digital communications [J]. IEEE trans. signal processing, 2002, 50(2): 255-270.

[9] FARHANG-BOROUJENY B, ZHU H, SHI Z. Markov chain Monte Carlo algorithms for CDMA and MIMO communication systems[J]. IEEE trans. signal processing, 2006, 54(5): 1896-1909.

[10] MACKAY D J C. Information theory, inference, and learning algorithms[M]. Cambridge: Cambridge University Press, 2003.

[11] KLEIN P. Finding the closest lattice vector when it's unusually close[C]/Proc of Eleventh Annual ACM-SIAM Symposium on Discrete Algorithms, San Francisco, California, USA, 2000:937- 941.

[12] LIU S, LING C, STEHLE D. Decoding by sampling: a randomized lattice algorithm for bounded distance decoding[J]. IEEE trans. inform. theory, 2001, 57(9): 5933-5945.

[13] BAI L, CHOI J. Lattice reduction-based mimo iterative receiver using randomized sampling[J]. IEEE trans. wireless communications, 2013, 12(5): 2160-2170.

[14] JALDEN J, SEETHALER D, MATZ G. Worst- and average-case complexity of LLL lattice reduction in MIMO wireless systems[C] //Proc of ICASSP, 2008: 2685- 2688.

[15] ANDERSON B D O, MOORE J B. Optimal filtering[M]. EnglewoodCliffs: Prentice-Hall, 1979.

\section{About the authors}

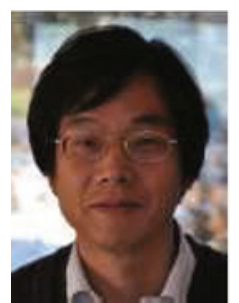

CHOI Jinho was b orn in Seoul, Korea. He received B.E. (magna cum laude) degree in electronics engineering in 1989 from Sogang University, Seoul, and the M.S.E. and Ph.D. degrees in electrical engineering from Korea Advanced Institute of Science and Technology (KAIST), Daejeon, in 1991 and 1994, respectively. He is with Gwangju Institute of Science and Technology (GIST) as a Professor. Prior to joining GIST in 2013, he was with the College of Engineering, Swansea University, United Kingdom, as a Professor/Chair in Wireless. His research interests include wireless communications and array/statistical signal processing. He authored two books published by Cambridge University Press in 2006 and 2010. Prof. Choi received the 1999 Best Paper Award for Signal Processing from EURASIP, 2009 Best Paper Award from WPMC (Conference), and is Senior Member of IEEE. Currently, he is an Associate Editor of IEEE COMMUNICATIONS LETTERS and an Editor of the Journal of Communications and Networks (JCN) since 2005 and served as an Associate Editor of IEEE TRANSACTIONS ON VEHICULAR TECHNOLOGY from 2005 to 2007 and ETRI journal. (Email: jchoi0114@gist.ac.kr)

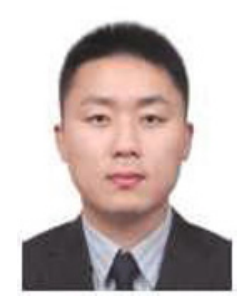

BAI Lin [corresponding author] received the B.Sc. degree in Electronics and Information Engineering from Huazhong University of Science and Technology, Wuhan, China, in 2004, the M.Sc. (with distinction) degree in communication systems from the University of Wales, Swansea, U.K., in 2007, and the Ph.D. degree in Advanced Telecommunications from the School of Engineering, Swansea University, U.K., in 2010. He is currently with the School of Electronics and Information Engineering, Beihang University (Beijing University of Aeronautics and Astronautics, BUAA), Beijing, China, as an Associate Professor. He is the author of the book Low Complexity MIMO Detection published by Springer in 2012. His research interests include signal processing of wireless communications, particularly multiple-input-multiple-output systems, array/smart antenna, and lattice-based approaches. Dr. Bai received an IEEE COMMUNICATIONS LETTERS Exemplary Reviewers Certificate for 2012. He is a Guest Editor of the International Journal of Distributed Sensor Networks since 2012. (Email: 1.bai@buaa edu.cn) 